\title{
The chimeric mice derived from umbilical cord blood stem cells of EGFP-transgenic mouse
}

\author{
Biao Duan, Haiyan Du, Rong Zhang \\ Reproductive Center, Department of Obstetric and Gynecology, The Third Affiliated Hospital of Inner Mongolia Medical \\ University, Baotou, China
}

Received: March 15, 2016

Accepted: April 21, 2016

Online Published: June 10, 2016

DOI: $10.14725 /$ dcc.v3n2p1

URL: http://dx.doi.org/10.14725/dcc.v3n2p1

\begin{abstract}
Objective: The chimeric mice were prepared by microinjection of blastocyst cavity using umbilical cord blood stem cells (UCBSCs) of Enhanced Green Fluorescent Protein (EGFP)-transgenic mouse, which was expected to provide a theoretical and experimental basis for the study of in-vivo differentiation of adult stem cells.

Methods: Mouse UCBSCs expressing green fluorescence was microinjected into blastocyst cavity and several blastocysts were transferred into uterus of pseudo pregnant mouse. First of all, new-born candidate chimeric mice were observed through feather color. Secondly, the genomic DNA and total RNA were extracted to analyze chimeric rate in several tissues. Finally, flow cytometry was used to detect percentage of green fluorescent cells mice in several tissues.

Results: The UCBSCs expressing green fluorescent protein were successfully isolated. After flow cytometry analysis, the proportion of cells expressing green fluorescence was $80.25 \%$. Through microinjection and embryo transfer, we got five white new-born mice and no chimeric feather color was observed. The analyses of PCR and RT-PCR were carried out to detect EGFP gene using six tissues including heart muscle, liver, lung, skin, leg muscle and adipose tissue. The results showed that the leg muscle and adipose tissue of two mice were positive and the other tissues and six tissues of the other 3 mice were all negative. The leg muscle and adipose tissue of two positive mice were digested into single-cells suspension and were carried out flow cytometry analysis. The results showed that the average chimeric rates of leg muscle and adipose tissue of two positive mice were $9.87 \%$ and $5.78 \%$, respectively.

Conclusions: The results demonstrated that adult UCBSCs could differentiate into leg muscle and adipose tissue in vivo.
\end{abstract}

Key Words: Cord blood stem cell transplantation, Green fluorescent proteins, Chimera, Mice, Cell differentiation

Embryonic stem cells are considered as pluripotent cells and can differentiate into three germ layers. ${ }^{[1,2]}$ Each germ layer will be differentiated into various tissues and organs of the organism. Adult stem cells are a type of multi-directional differentiation potential cells. Umbilical cord blood stem cells (UCBSCs) are one of the most studied adult stem cells. It has many advantages, such as extensive sources, abundant contents, convenient acquisition, strong differentiation and stable biological properties. It has broad application prospects in the field of stem cell therapy. UCBSCs have been demonstrated in many experiments to have the potential to differentiate into any tissue of interstitial potential, such as fat cells, ${ }^{[3]}$ liver cells, ${ }^{[4]}$ muscle cells, ${ }^{[5]}$ nerve cells ${ }^{[6]}$ and so on. However, studies on the differentiation of UCB cells have focused on the induction of differentiation into specific types of cells in vitro. In vivo, the studies focused on the differentiation of adult stem cells into specific lesions and the differentiation of cells at specific sites. ${ }^{[7]}$ However, it only detects the ability of adult stem cells to develop in a certain direction, which makes the method lim-

*Correspondence: Haiyan Du; E-mail: 13947268268@139.com; Address: Reproductive Center, Department of Obstetric and Gynecology, The Third Affiliated Hospital of Inner Mongolia Medical University, Baotou, China. 
ited.

In general, methods for the preparation of chimeric animals by microinjection into the blastocoel are used to detect the developmental potential of embryonic stem cells. Adult stem cells, unlike the omnipotent stem cells of embryonic stem cells, can also be induced in vitro to differentiate into cells of any tissue derived from the mesenchymal origin. In this study, we used embryonic stem cells to study the differentiation of UCBSCs, and made the chimera animal with microinjection of blastocyst with green fluorescence, so as to study the differentiation of adult stem cells in vivo.

\section{Materials and method}

\subsection{Materials}

\subsubsection{Experimental reagents}

PMSG and hCG were produced by Ningbo Sansheng Pharmaceutical Co., Ltd.; DMEM, standard fetal bovine serum, penicillin streptomycin antibiotics, $0.25 \%$ trypsin were purchased from Gibco; Ficoll lymphocyte separation [density $(1.0770 \pm 0.0001) \mathrm{g} / \mathrm{ml}$ ] was purchased from Tianjin Hao Yang Biological Products Science and Technology Co., Ltd.; KSOM and M2 medium were both Japanese and light industrial products. Whole genome extraction kit, reverse transcription RT-PCR detection kit, Taq DNA polymerase were purchased from Dalian Bao Biological Company, PCR primers were synthesized by Beijing Invitrogen company.

\subsubsection{Experimental animals}

Enhanced green fluorescent protein (EGFP)-transgenic mice (from C57BL/6J) and Kunming white mice were purchased from Experimental Animal Research Center of Inner Mongolia University.

\subsection{Method}

\subsubsection{Isolation of UCBSCs of EGFP-transgenic mice}

The female and male EGFP-transgenic mice of 8 weeks old were fed with a proportion of 1: 1 in each cage, and the female mice were fed separately on the next day if vaginal suppositories were detected. In the full term, the blood of the newborn mice was extracted and placed in the anticoagulant centrifuge tube of $10 \mathrm{ml}$. Blood mononuclear cells were isolated using a modified density gradient method. Anticoagulant cord blood was added to the same volume of PBS dilution with a proportion of 1: 1, and mixed. $5 \mathrm{ml}$ of diluted umbilical cord blood was slowly added to $5 \mathrm{ml}$ of Ficoll Lymphocyte Separation Solution and centrifuged at $1,600 \mathrm{r} / \mathrm{min}$ for $15 \mathrm{~min}$ at room temperature. The mononuclear cell layer in the middle white to $5 \mathrm{ml}$ of DMEM con- taining $10 \%$ fetal bovine serum was gently taken and mixed and centrifugated at 1,200 r/min at room temperature for 8 min. After counting, the cells were seeded at a density of $2 \times 10^{5}$ cells $/ \mathrm{cm}^{2}$ in $5 \mathrm{ml}$ of DMEM containing $10 \%$ fetal bovine serum in a $60 \mathrm{~mm}$ Petri dish at $37^{\circ} \mathrm{C}, 5 \% \mathrm{CO}_{2}, 100 \%$ humidity for mononuclear cells.

\subsubsection{Generation and preparation of genetically modi- fied UCBSCs}

After $48 \mathrm{~h}$, the culture medium was replaced and the liquid was changed every other day. The cells adherence and growth were observed under the inverted phase contrast microscope. When cells growed to $90 \%$ confluency, the $0.25 \%$ pancreatin was digested by $1: 2$.

According to the concentration of cells, some cells were under frozen storage, and some continued to passage. $1 \mathrm{ml}$ medium was added to the culture dish, in which few were remained, by gently pipetting minor adhesion in UCBSCs of Petri dish. Transferred the liquid to $1.5 \mathrm{ml} \mathrm{EP}$ tube, and stored at $4^{\circ} \mathrm{C}$ for later use.

\subsubsection{Embryos collection and blastocyst preparation}

The female and male Kunming mice of 8 weeks old were fed with a proportion of 1: 1 in each cage, and the female mice were fed on the next day if vaginal suppositories were detected. After seeing the bolt for 3.5 days, the uterus of mice was completely removed and placed on a sterile Petri dish when the vaginal embolus or sperm in vaginal cells smear was inspected. The uterus was cut in half along the cervix, with $1 \mathrm{ml}$ syringe to absorb M2 liquid to carry out embryos, respectively. The blastocysts and morulae were collected by microscopic examination. After washing with KSOM medium for three times, they were placed in pre-equilibrated $\mathrm{KSOM}$ solution and cultured under the conditions of $37^{\circ} \mathrm{C}$, $5 \% \mathrm{CO}_{2}$ and $100 \%$ humidity. The development of blastocyst was observed and recorded.

\subsubsection{Blastocyst microinjection and embryo transfer}

Ten green fluorescent-emitting mesenchymal stem cells were injected into the expanded blastocoel by microinjection under a blue light-activated fluorescence microscope. The mesenchymal stem cells were gently blown out so that they just fell on the inner cell mass, and then the injection needle was quickly pulled out. After injection, the blastocyst cavity disappeared and was then washed with KSOM medium for three times, placed in new KSOM culture medium and resumed blastocyst cavity after incubating at $37^{\circ} \mathrm{C}, 5 \% \mathrm{CO}_{2}$ and $100 \%$ humidity for $1-2 \mathrm{~h}$. 


\subsubsection{Embryo transfer}

The adult Kunming white female mice of superovulation and the ligation of Kunming male mice were fed with a proportion of 1: 1 in each cage and vaginal suppositories were inspected on the following day. On the $0.5 \mathrm{~d}$, the female mice were fed alone once they were identified as pseudopregnancy, and treated with embryo transfer on the $2.5 \mathrm{~d}$ when vaginal embolus was observed.

A blunt syringe of $1 \mathrm{ml}$ was placed on the corner of the uterus wall with a needle. The $7 / 8$ embryo sucking needles were inserted into the uterus in the direction of the cervix, and the embryo was blown into the uterus. After suture of abdominal wall and skin, the female mice were placed into the cage for later delivery.

\subsubsection{Analysis of mice chimerism}

The leg muscles, myocardium, liver, lung, skin and adipose tissues of the chimeric mice to be identified and the Kunming white mice of the same age (as a control) were collected. The genomic DNA and total RNA of each tissue were extracted, PCR and RT-PCR were taken with EGFP specific primers $\mathrm{P} 1$ and $\mathrm{P} 2$ to identify the presence and expression of chimeric cells of EGFP gene in different tissues.

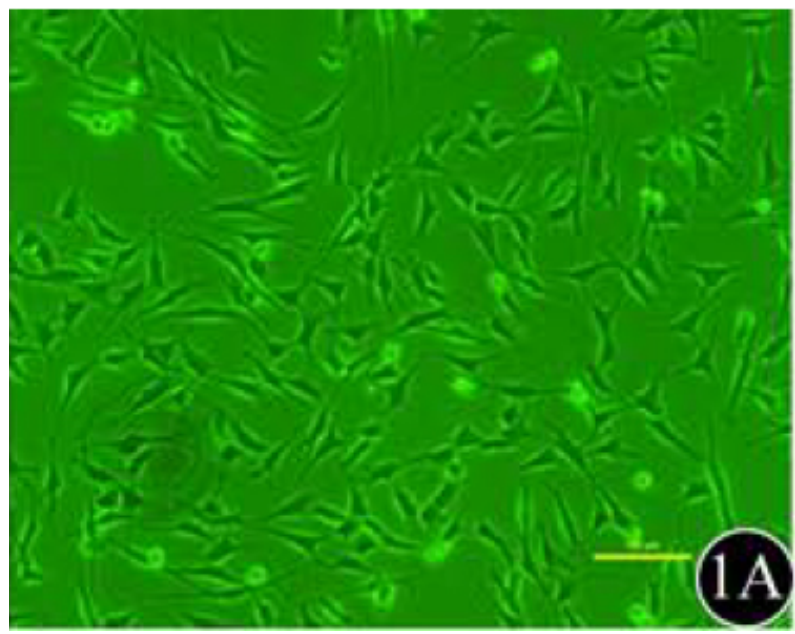

The $\beta$-action was selected as a reference gene. Primer sequences were: P1: 5'-CAGTGCTTCAGCCGCTACCC-3'; P2: 5'-AGTTCACCTTGATGCCGTTCTT-3'. The reaction conditions were $95^{\circ} \mathrm{C}$ for $3 \mathrm{~min}$ pre-denaturation followed by 30 cycles of last extension at $94^{\circ} \mathrm{C}$ for $40 \mathrm{~s}, 61^{\circ} \mathrm{C}$ for $20 \mathrm{~s}, 72^{\circ} \mathrm{C}$ for $30 \mathrm{~s}, 72^{\circ} \mathrm{C}$ for $10 \mathrm{~min}$. The PCR product was expected to be $289 \mathrm{bp}$ in size.

\subsubsection{Flow cytometry}

The leg muscles, heart, kidney and adipose tissues of two chimeric mice were cut, trypsin digestion for $25 \mathrm{~min}$, terminated with PBS solution containing $10 \%$ fetal bovine serum, syringe and percussion until the liquid turbidity. The absorbed digestive fluid was filtered into a centrifuge tube with 100 mesh stainless steel filter. Centrifuged at 1,500 $\mathrm{r} / \mathrm{min}$ at room temperature for $5 \mathrm{~min}$. Discarded the supernatant, added $1 \mathrm{ml}$ of PBS and used a pipette tip to mix well. Next, centrifuged at 3,000 r/min using Percoll density gradient centrifugation for $10 \mathrm{~min}$. After centrifugation, aspirated the cell layer into an Eppendorf tube containing $1 \mathrm{ml}$ of PBS solution and blew the cell suspension with a pipette tip. Detection was performed using a Beckman \& Coulter Cell Lab Quanta SC flow cytometer.

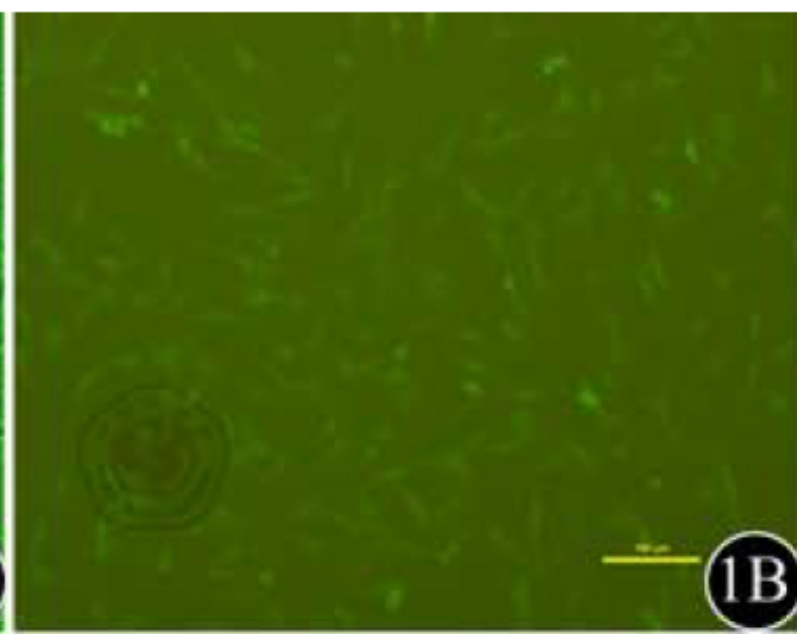

Figure 1: UCBSCs of EGFP-transgenic mouse. 1A: Phase contrast microscope photograph; 1B: Fluorescence excitation photograph with $100 \mu \mathrm{m}$ scale

\section{Results}

\subsection{Acquisition of UCBSCs of EGFP-transgenic mouse}

Cell adherence was observed on the $3 \mathrm{~d}$, mainly with multinuclear and large megakaryocytes and mononuclear spindle shaped fibroblast like cells.

Fibroblast-like cells formed colonies in about 10 days, and the confluence of fibroblast-like cells reached about $80 \%$ af- ter about 20 days (see Figure 1A). Under fluorescence microscope, green fluorescence was observed (see Figure 1B).

\subsection{Preparation of chimeric mice}

A total of 29 embryos were transplanted into the uterus of 3 pseudopregnant female mice when blastocyst cavity recovered from microinjection. 5 little mice were born at fullterm by one pseudo-pregnant rat after $19.5 \mathrm{~d}$ of pregnancy. 


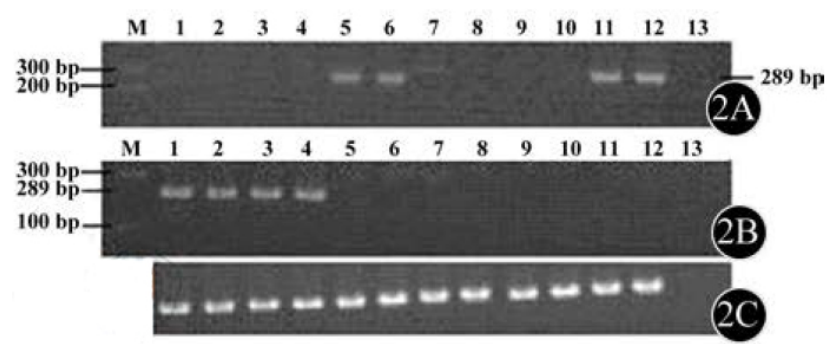

Figure 2: The PCR and RT-PCR electrophoresis results of Q-1 and Q-2 mice transfected with EGFP gene chimerism. 2A: PCR electrophoresis, $\mathrm{M}$ is the marker, genomic DNA shown in lanes 1-6 is from Q-1, including myocardial, liver, lung, skin, leg muscle and fat respectively; genomic DNA shown in lanes 7-12 is from Q-2, including myocardial, liver, lung, skin, leg muscle and fat respectively; lane 13 is for $\mathrm{ddH}_{2} \mathrm{O} ; 2 \mathrm{~B}$ : RT-PCR electrophoresis, $\mathrm{M}$ is the marker; lanes 1-4 is for thigh muscles and fat of Q-1 and Q-2 respectively; lanes 5-12 is for heart, lung, liver and skin of Q-1 and Q-2; lane 13 is for $\mathrm{ddH}_{2} \mathrm{O} ; 2 \mathrm{C}$ : RT-PCR electrophoresis of $\beta$-actin, the sequence of lanes 1-13 is the same as those in Figure 2B

\subsection{Identification of mice transfected with EGFP gene chimeras}

No hair color mosaic was observed in the 5 newborn mice, all of which were white. In order to identify whether the chimera animals were obtained, five mice were killed by cervical dislocations, and tissues including myocardium, liver, lung, skin, leg muscle and fat were collected. The genomic DNA was first extracted for PCR reaction. The leg muscles and adipose tissue of 2 mice were positive (see Figure 2A), and the other 4 tissues were negative. The two mice were named Q-1 and Q-2. The total RNA from 6 tissues of Q-1 and Q-2 was extracted and subjected to RT-PCR. The results showed that only leg muscle and adipose tissue were positive (see Figure 2B and 2C), and the other 4 tissues were negative.

\subsection{Flow cytometric analysis}

The thigh muscle and adipose tissue of two chimeric mice were digested into single cell suspensions, respectively, and the normal muscles of the Kunming white mice (as a negative control) and the isolated EGFP gene UCBSCs (as a positive control) were subjected to flow cytometry together. As a result, no green fluorescence in the leg muscles was observed in normal Kunming white mice (see Figure 3A), and the proportion of green fluorescent cells in the separated cord blood stem cells was $80.25 \%$ (see Figure 3B), chimeric rates of leg muscle and fat in Q-1 were $10.52 \%$ and $6.23 \%$, respectively (see Figure $3 \mathrm{C}$ and $3 \mathrm{E}$ ). The chimeric rates of leg muscle and fat were $9.21 \%$ and $5.33 \%$, respectively (see
Figure 3D and 3F). The average chimeric rates were $9.87 \%$ and $5.78 \%$ respectively. There was no chimerism in the renal and myocardial tissues of Q-1 and Q-2 (see Figure 3G to $3 \mathrm{~J}$ ). The group diagram of the flow cytometry was shown in Figure 3.

\section{Discussion}

UCBSCs have strong self-renewal and multi-directional differentiation ability. In vitro induction of differentiation, it can differentiates into osteoblasts, muscle cells and adipocytes. Some experiments have shown that the proliferation and differentiation of UCBSCs is stronger than that of bone marrow mesenchymal stem cells and adipose-derived mesenchymal stem cells. ${ }^{[8]}$

Blastocysts microinjection is often used to study omnipotent developmental ability of embryonic stem cells. Microinjection has become an important method to study the differentiation of embryonic stem cells in vitro, especially gametes, since Bradley et al. ${ }^{[9]}$ successfully obtained germline chimeric mice using this method in 1984. Adult stem cells are less advanced than embryonic stem cells in developmental potential, but they also have pluripotency. In this experiment, we used a microinjection method to study the in-vivo differentiation of UCBSCs. All 5 newborn mice were white, with no hair color chimerism. This showed that cells from adult cord blood stem cells did not differentiate into skin tissue cells. Dermal tissue is derived from ectodermal differentiation. Embryonic stem cells have the ability to differentiate into three germ layers. All the chimeric animals have hair color chimerism, but the differentiation ability of adult stem cells is limited. ${ }^{[10]}$ It only differentiates into cells derived from mesenchymal cells or tissues, which may partly explain why there is no coiffure on the hair color. To further determine whether these pups were chimeric animals, five mice were sacrificed by cervical dislocation, and six tissues including myocardium, liver, lung, skin, leg muscle and fat were taken. Genomic DNA and total RNA were successively extracted for PCR and RT-PCR reactions. The primers were designed based on the green fluorescent protein gene fragment. Only two mice were identified as positive for leg muscle and adipose tissue and the other four were negative. These results indicated that these two mice had chimerism in adult leg muscle and adipose tissue. Adult UCBSCs could differentiate into muscle and adipose tissue in vivo, which is consistent with the results of induced differentiation in vitro. ${ }^{[11,12]}$ Next, in order to further investigate the degree of green fluorescent cells chimerism in the leg muscle and adipose tissue, we made the tissue into a single cell suspension using a combination of trypsinization and mechanical pipetting, followed by flow cytometry. The results showed that the average chimeric rates of two chimeric mice in leg muscle and adipose tissue were 9.87\% and $5.78 \%$ respectively. These results showed that mouse 
UCBSCs obtained chimerism in the leg muscles and adi- hair color chimerism was observed. pose tissue while expressing green fluorescence, though no
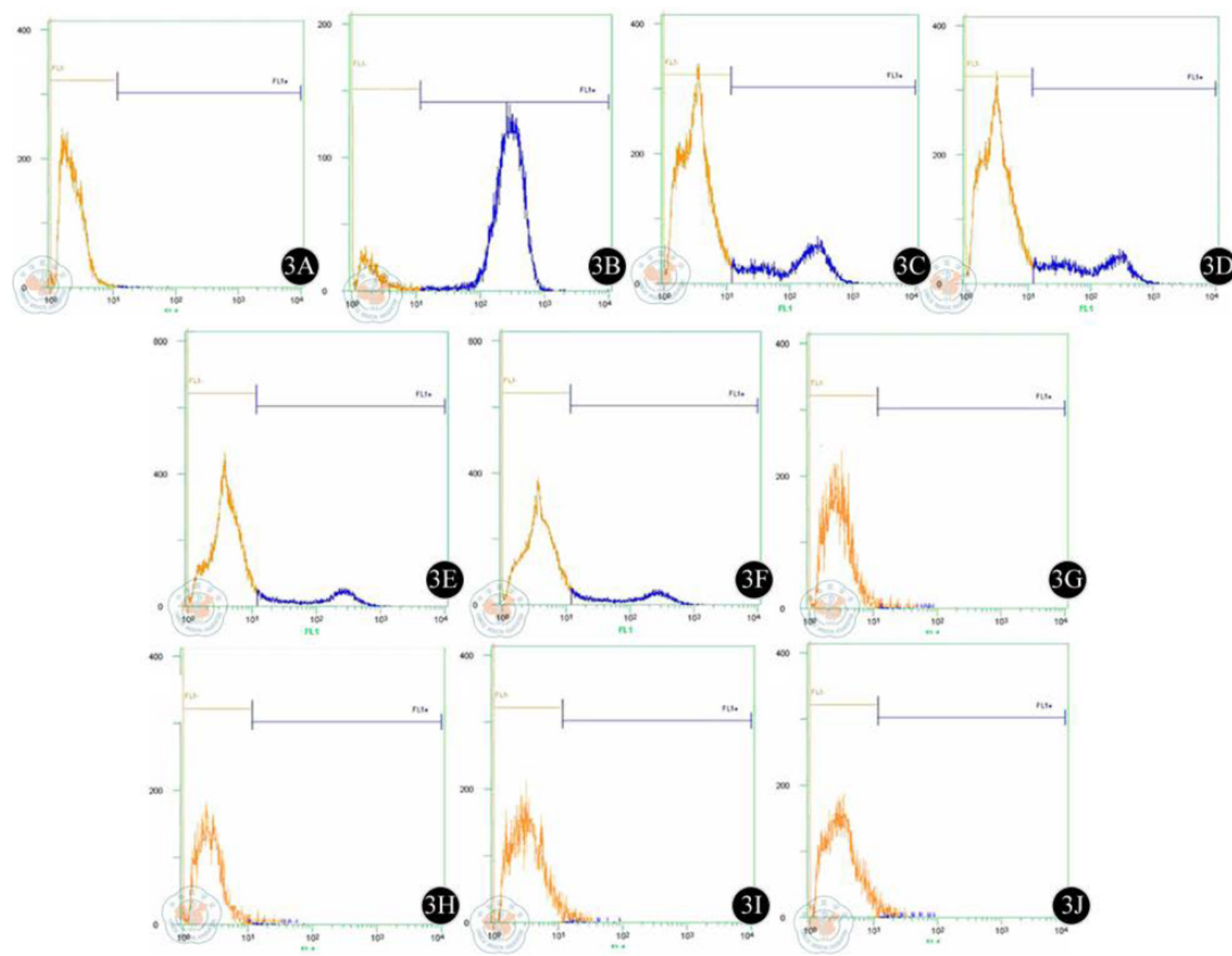

Figure 3: Group diagrams of flow cytometry. 3A: Leg muscle of normal Kunming white mouse; 3B: Cord blood stem cell transfected with EGFP gene; 3C: Q-1 leg muscle; 3D: Q-2 leg muscle; 3E: Q-1 adipose tissue; 3F: Q-2 adipose tissue; 3G: Q-1 kidney tissue; 3H: Q-2 kidney tissue; 3I: Q-1 myocardial tissue; 3J: Q-2 myocardial tissue

\section{Conclusions}

In conclusion, adult UCBSCs, after microinjection, can play an important role in cell differentiation in vivo with intracellularized lump cells. These experimental studies may provide a new idea for studying the differentiation of adult stem cells in vivo.

\section{Conflicts of Interest Disclosure}

The authors have no conflicts of interest related to this article.

\section{References}

[1] Nakano T, Kodama H, Honjo T. Generation of lymphohematopoietic cells from embryonic stem cells in culture. Science. 1994; 265: 1098-1101. PMid: 8066449. https://doi.org/10. 1126 /science. 8066449

[2] Brustle O, Jones KN, Learish RD, et al. Embryonic stem cellderived glial precursors: a source of myelinating transplants. Sci-

ence. 1999; 285: 754-756. PMid: 10427001. https://doi.org/ $10.1126 /$ science 285.5428 .754

[3] Qu CQ, Zhang GH, Zhang LJ, et al. Osteogenic and adipogenic potential of porcine adipose mesenchymal stem cells. In Vitro Cell Dev Biol Anim. 2007; 43: 95-100. PMid: 17570023. https: //doi.org/10.1007/s11626-006-9008-y

[4] Jung YJ, Ryu KH, Cho KA, et al. In vitro hepatic differentiation of human umbilical cord blood and bone marrow cells. Pedi- 
atr Hematol Oncol. 2008; 25: 481-491. PMid: 18728967. https : //doi.org/10.1080/08880010802234614

[5] Kadner A, Hoerstrup SP, Tracy J, et al. Human umbilical cord cells: a new cell source for cardiovascular tissue engineering. Ann Thorac Surg. 2002; 74: S1422-1428. https://doi.org/10.1016/ S0003-4975 (02) 03910-3

[6] Jin GZ, Yin XJ, Yu XF, et al. Generation of neuronal-like cells from umbilical cord blood-derived mesenchymal stem cells of a RFPtransgenic cloned cat. J Vet Med Sci. 2008; 70: 723-726. PMid: 18685247. https://doi.org/10.1292/jvms.70.723

[7] Li HJ, Liu HY, Zhao ZM, et al. Transplantation of human umbilical cord stem cells improves neurological function recovery after spinal cord injury in rats. Zhongguo Yi Xue Ke Xue Yuan Xue Bao. 2004; 26: 38-42. PMid: 15052772.

[8] Kern S, Eichler H, Stoeve J, et al. Comparative analysis of mesenchymal stem cells from bone marrow, umbilical cord blood, or adipose tissue. Stem Cells. 2006; 24: 1294-1301. PMid: 16410387. https://doi.org/10.1634/stemcells.2005-0342
[9] Bradley A, Evans M, Kaufman MH, et al. Formation of germ-line chimaeras from embryo-derived teratocarcinoma cell lines. Nature. 1984; 309: 255-256. PMid: 6717601. https://doi.org/10. $1038 / 309255 \mathrm{aO}$

[10] Chamberlain G, Fox J, Ashton B, et al. Concise review: mesenchymal stem cells: their phenotype, differentiation capacity, immunological features, and potential for homing. Stem Cells. 2007; 25: 2739-2740. PMid: 17656645. https://doi.org/10.1634/ stemcells.2007-0197

[11] Pereira WC, Khushnooma I, Madkaikar M, et al. Reproducible methodology for the isolation of mesenchymal stem cells from human umbilical cord and its potential for cardiomyocyte generation. J Tissue Eng Regen Med. 2008; 21: 394-399. PMid: 18615777. https://doi.org/10.1002/term.107

[12] Wagner W, Wein F, Seckinger A, et al. Comparative characteristics of mesenchymal stem cells from human bone marrow, adipose tissue, and umbilical cord blood. Exp Hematol. 2005; 33: 1402-1416. PMid: 16263424. https://doi.org/10.1016/j. exphem.2005.07.003 